\title{
On the Adiabatic Limit for Dirac Particles in External Fields
}

\author{
G. Nenciu* \\ Institut für Theoretische Physik der Universität Zürich, CH-8001 Zürich, Switzerland
}

\begin{abstract}
An adiabatic switching formalism is proposed to bypass the difficulties in defining the spontaneous pair creation in static electromagnetic fields.
\end{abstract}

\section{Introduction}

The interest in the basic theoretical problems of Q.E.D. (Quantum ElectroDynamics) in strong external electromagnetic fields has been stimulated in the last years by the experimental facilities of the heavy ion physics. During the collision of two heavy nuclei a very strong electromagnetic field is created and new phenomena, absent in weak fields, are expected to arrise.

From the experimental point of view, the most striking phenomenon which is expected to set in is the "spontaneous" creation of electron-positron pairs.

A rich literature exists [1-4] concerning Q.E.D. in strong fields, but most of the results are based on nonrigorous treatments, analogies and speculations. In particular, the mechanism of spontaneous pair creation is explained usually [3] by a "gedanken" experiment as an adiabatic increase of the external charge, via the analogy with selfionization of helium. In our opinion, it is very hard to put these calculations on firm basis without relying on scattering theory. In fact the pair creation, spontaneously or not, is a scattering phenomenon and must be treated as such.

The main difficulty in the subject is the fact that one cannot use perturbation theory with respect to the external field. On the other hand, if one neglects the electromagnetic field created by the electron-positron field, the problem can be treated by nonperturbative methods. This approximation is known as the external field problem in Q.E.D. and there exist a lot of rigorous result about it (see $[5,6]$ and references therein, also [7-10] for recent results; for a review of rigorous results, relevant to strong field phenomena see [11]).

Unfortunately, the discussion of the problem of spontaneous pair creation, in the framework of the external field problem in Q.E.D., meets some difficulties

* On leave from I.F.I.N. Bucharest, Rumania 
because of the result of Bongaarts [12] that static fields are unable to create pairs. (This fact has been noted earlier in the literature, see for example [13].)

The aim of this paper is to propose a new way of dealing with this problem, in the framework of the adiabatic switching formalism. In Sect. 2, the problem is described, the notions of "undercritical" and "overcritical" static fields are made precise, and some conjectures are put forward. In Sect. 3, one of the conjectures, concerning undercritical fields is proved.

\section{Description of the Problem}

Since all results of the external field problem in Q.E.D. can be obtained from results about the classical Dirac equation via the machinery of second quantization, we shall start by fixing some notation for the Dirac equation.

The Dirac equation with time dependent potentials reads

$$
i \frac{d}{d t} \psi_{t}(\mathbf{x})=\left(-i \alpha \nabla+\beta m+\mathbf{V}_{t}(\mathbf{x})\right) \psi_{t}(\mathbf{x})
$$

the underlying Hilbert space being $\mathscr{L}^{2}\left(\mathbb{R}^{3}\right)=\left(L^{2}\left(\mathbb{R}^{3}\right)\right)^{4}$ and

$$
\mathbf{V}_{t}(\mathbf{x})=-e \phi(t, \mathbf{x})+e \alpha A(t, \mathbf{x})
$$

$\phi$ and $A$ are the electric and magnetic potentials, respectively. By $\mathbf{H}_{0}$ we shall denote the free Dirac operator which is the selfadjoint extension of $(-i \alpha \nabla+\beta m)$ in $\mathscr{L}^{2}\left(\mathbb{R}^{3}\right)$. In what follows, we shall assume that for all $t \in \mathbb{R}, \mathbf{V}_{t}$ is $\mathbf{H}_{0}$-bounded with relative bound less than one so that $\mathbf{H}_{t}=\mathbf{H}_{0}+\mathbf{V}_{t}$ is self-adjoint on $\mathscr{D}\left(\mathbf{H}_{0}\right)$ by the Rellich-Kato theorem [14]. For a given potential $\mathbf{V}$ (not necessarily time independent) $S(\mathbf{V})$ is the scattering operator associated to the pair $\mathbf{H}_{0}$, $\mathbf{H}_{t}=\mathbf{H}_{0}+\mathbf{V}_{t}$.

Finally let $P_{\sigma}^{0}, \sigma= \pm$ be the spectral projections of $\mathbf{H}_{0}$ corresponding to $[m, \infty)$ and $(-\infty,-m]$ respectively.

The following results will be relevant for our discussion.

Theorem 1. Suppose that $\mathbf{V}$ is time independent, $\mathbf{H}_{0}$-bounded with relative bound less than one and that $S(\mathbf{V})$ exists and is unitary. Then

i) for $\sigma \neq \sigma^{\prime}$

$P_{\sigma}^{0} S(\mathbf{V}) P_{\sigma^{\prime}}^{0}=0$.

ii) Let $\mathbf{V}_{\varepsilon}(t)=\exp \left(-\varepsilon^{2} t^{2}\right) \mathbf{V}$. Then $S\left(\mathbf{V}_{\varepsilon}\right)$ exists is unitary and for all $f, g \in \mathscr{L}^{2}\left(\mathbb{R}^{3}\right)$

$$
\lim _{\varepsilon \rightarrow 0}\left(f, S\left(\mathbf{V}_{\varepsilon}\right) g\right)=(f, S(\mathbf{V}) g) .
$$

Remark 1. The results stated in Theorem 1 are usually proved in the Schrödinger case, but it is easy to see that the proofs are the same in the Dirac case. The first one is based on the well-known fact that the scattering operator commutes with the free evolution. The second one is due to Dollard [15] and says that the adiabatic theorem for the scattering operator is true in the weak topology. 
Let now $\mathscr{F}_{0}$ be the Cook-Fock space for the free electron-positron field. One of the basic results of the quantized theory is $[5,6,16]$

Theorem 2. Suppose that $S(\mathbf{V})$ exists and is unitary. Then

i) the scattering theory of the quantized Dirac field in the external field $\mathbf{V}$ is described by a unitary operator $\mathscr{S}(\mathbf{V})$ in $\mathscr{F}_{0}$ if and only if $P_{\sigma}^{0} S(\mathbf{V}) P_{\sigma^{\prime}}^{0}$ is HilbertSchmidt for $\sigma \neq \sigma^{\prime} . \mathscr{S}(\mathbf{V})$ is uniquely determined by $S(\mathbf{V})$ up to a phase factor.

ii) The phase in $\mathscr{S}(\mathbf{V})$ can be chosen such that, if $\Omega$ is the vacuum state in $\mathscr{F}_{0}$, then

$$
(\Omega, \mathscr{S}(\mathbf{V}) \Omega)= \begin{cases}0 \quad \text { if } A A^{*} & \text { has } 1 \text { as one of its eigenvalues } \\ \operatorname{det}\left(1+B A A^{*} B^{*}\right)^{-1 / 2} & \text { otherwise, }\end{cases}
$$

where

$$
A=P_{+}^{0} S(\mathbf{V}) P_{-}^{0} ; B=\left(P_{+}^{0} S(\mathbf{V}) P_{+}^{0}\right)^{-1} .
$$

The formula (2.5) is the staring point of our discussion. Since $1-(\Omega, \mathscr{S}(\mathbf{V}) \Omega)^{2}$ is the total probability of pair creation, (2.5) combined with Theorem 1 i) shows that there is no pair creation in a static field of arbitrary strength. The inability of a static field to create pairs has been noted early [13], and proved rigorously by Bongaarts [12] in the $C^{*}$-algebra framework. However, this result does not forbid the "spontaneous" pair creation (as one could think at the first sight). The question is whether the static field result is stable in the following sense. Suppose $\mathbf{V}$ is not strictly time independent but varies very slowly in time. Is it then true that $(\Omega, \mathscr{S}(\mathbf{V}) \Omega) \simeq 1$ ? If the answer is negative, one shall say that a spontaneous pair creation sets in.

The above idea can be formulated precisely in the framework of the adiabatic limit. Let $\mathbf{V}$ be a time independent field, and $\mathbf{H}_{\varepsilon}=\mathbf{H}_{0}+\exp \left(-\varepsilon^{2} t^{2}\right) \mathbf{V}$ with $S\left(\mathbf{V}_{\varepsilon}\right)$ the corresponding scattering operator.

Definition 1. a) A time independent external field $\mathbf{V}$ is said to be undercritical if

$$
\lim _{\varepsilon \rightarrow 0}\left(\Omega, \mathscr{S}\left(\mathbf{V}_{\varepsilon}\right) \Omega\right)=1 \text {. }
$$

b) A time independent external field $\mathbf{V}$ is said to be overcritical if

$$
\lim _{\varepsilon \rightarrow 0}\left(\Omega, \mathscr{S}\left(\mathbf{V}_{\varepsilon}\right) \Omega\right)<1 .
$$

In the rest of this section $\mathbf{V}$ will be considered $\left\{\left.\mathbf{H}_{0}\right|^{\alpha}\right.$ compact for some $\alpha<1$.

Let $\mathbf{V}(\mathbf{x})=V(\mathbf{x}) \mathbb{1}, V(\mathbf{x})<0$, i.e. $\mathbf{V}$ is the external field created by a static distribution of positive charges, $\mathbf{V}_{\lambda}=\lambda \mathbf{V}, \lambda \geqq 0$ and $\mathbf{H}_{\lambda}=\mathbf{H}_{0}+\mathbf{V}_{\lambda}$. It is believed [11] that when $\lambda$ increases from 0 to $\infty$ eigenvalues appear at $+m$, moves continuously to the left and dive in the negative continuum at $-m$. Let $\lambda_{c}$ be the value of $\lambda$ when the first eigenvalue reaches $-m$. The common belief among physicists amounts for the following:

Conjecture 1. $\mathbf{V}_{\lambda}$ is undercritical for $\lambda<\lambda_{c}$ and overcritical for $\lambda>\lambda_{c}$. More precisely:

$$
\lim _{\varepsilon \rightarrow 0}\left(\Omega, \mathscr{S}\left(\mathbf{V}_{\lambda, \varepsilon}\right) \Omega\right)=\left\{\begin{array}{lll}
1 & \text { for } & \lambda<\lambda_{c} \\
0 & \text { for } & \lambda>\lambda_{c} .
\end{array}\right.
$$


The restriction to purely electrical fields is not essential and we shall always consider general electromagnetic fields in the following.

Conjecture 2. Let $\mathbf{V}$ be time independent, and $\mathbf{H}_{s}=\mathbf{H}_{0}+\exp \left(-s^{2}\right) \mathbf{V}$. Suppose that there exist a continuous function $f(s), \lim _{s \rightarrow \pm \infty} f(s)=0$ and a constant $d>0$, such that the spectrum $\Sigma(s)$ of $\mathbf{H}_{s}$ has the property:

$$
\begin{aligned}
& \Sigma(s)=\Sigma_{+}(s) \cup \Sigma_{-}(s) ; \\
& \Sigma_{+}(s) \subset[f(s)+d / 2, \infty), \Sigma_{-}(s) \subset(-\infty,-d / 2+f(s)] .
\end{aligned}
$$

Then $\mathbf{V}$ is undercritical.

Roughly speaking, Conjecture 2 says that a static external field is undercritical if during the switching on and off, the spectrum emerging from the upper continuum does not mix with the spectrum emerging from the lower continuum. The above conjecture says nothing about the case when during the switching some eigenvalues emerging from the lower continuum cross some eigenvalues emerging from upper continuum. The most general conjecture is the following.

Conjecture 3. A static field is undercritical if and only if during the switching the eigenvalues emerging from the upper continuum does not touch the lower continuum and the eigenvalues emerging from the lower continuum does not touch the upper continuum.

In the following section, we shall prove Conjecture 2. In fact our method combined with the adiabatic theorem for intersecting eigenvalues $[17,18]$ can be used to prove the "if" part of the Conjecture 3. But the important problem which is left open is the existence of overcritical fields.

\section{The Results}

Definition 2. $\varphi(s), s \in \mathbb{R}$ is said to be a switching factor if

i) $0 \leqq \varphi(s) \leqq 1, \lim _{s \rightarrow 0} \varphi(s)=1, \lim _{s \rightarrow \pm \infty} \varphi(s)=0$.

ii) $\varphi(s)$ is twice differentiable on $\mathbb{R}$ and

$$
\begin{aligned}
& \sup _{s \in \mathbb{R}}\left|\frac{d}{d s} \varphi(s)\right|<\infty, \lim _{s \rightarrow \pm \infty} \frac{d}{d s} \varphi(s)=0, \\
& \int_{-\infty}^{\infty}\left(\left|\frac{d}{d s} \varphi(s)\right|^{2}+\left|\frac{d^{2}}{d s^{2}} \varphi(s)\right|\right) d s<\infty .
\end{aligned}
$$

The following is a trivial consequence of Definition 2.

Lemma 1. Let $\varphi(s)$ be a switching factor. Then

$$
\begin{aligned}
& \lim _{\varepsilon \rightarrow 0} \sup _{t \in \mathbb{R}}\left|\frac{1}{\varepsilon} \frac{d}{d t} \varphi(\varepsilon t)\right|<\infty, \\
& \lim _{\varepsilon \rightarrow 0}|\varepsilon|^{-1} \int_{-\infty}^{\infty}\left(\left|\frac{d}{d t} \varphi(\varepsilon t)\right|^{2}+\left|\frac{d^{2}}{d t^{2}} \varphi(\varepsilon t)\right|\right) d t<\infty .
\end{aligned}
$$


Remark 2. The usual switching factor $\exp (-\varepsilon|t|)$ is not contained in Definition 2, because the second derivative contains a $\delta$ function. But the reader will realise that all the proofs below can be extended to cover also this case.

The following result has a preparatory character.

Theorem 3. Let $\mathbf{H}_{0}$ be the free Dirac operator, $\mathbf{V}$ a time independent external field whose matrix elements $V_{i j}(\mathbf{x})$ are in $L^{p}\left(\mathbb{R}^{3}\right) \cap L^{2}\left(\mathbb{R}^{3}\right)$ for some $p>3$, and $\varphi(s)$ a switching factor. Then

i) for all $2 \geqq \alpha>3 / p$

$$
\left\|\left|\mathbf{H}_{0}\right|^{-\alpha} \mathbf{V}\right\|<\infty \text {. }
$$

In particular $\mathbf{V}$ is $\mathbf{H}_{0}$-compact and

$$
\lim _{a \rightarrow \infty}\left\|\left(\mathbf{H}_{0}-i a\right)^{-1} \mathbf{V}\right\|=0 \text {. }
$$

ii) For all $2 \geqq \alpha>3 / 2$

$$
\left\|\left|\mathbf{H}_{0}\right|^{-\alpha} \mathbf{V}\right\|_{\text {H.S. }}<\infty \text {, }
$$

where $\|\cdot\|_{\text {H.S. }}$ means the Hilbert-Schmidt norm.

iii) For all $\varepsilon, t \in \mathbb{R}, \mathbf{H}_{\varepsilon}(t)=\mathbf{H}_{0}+\varphi(\varepsilon t) \mathbf{V}$ is self-adjoint on $\mathscr{D}\left(\mathbf{H}_{0}\right)$.

iv) For all $\varepsilon, t_{0} \in \mathbb{R}$ the equation

$$
i \frac{d}{d t} U_{\varepsilon}\left(t, t_{0}\right)=\mathbf{H}_{\varepsilon}(t) U_{\varepsilon}\left(t, t_{0}\right) ; \quad U_{\varepsilon}\left(t_{0}, t_{0}\right)=1
$$

has a unique solution which is a strongly continuous family of unitary operators.

v) The Möller operators

$$
W_{\varepsilon}^{ \pm}=s-\lim _{t \rightarrow \pm \infty} U_{\varepsilon}^{*}(t, 0) \exp \left(-i t \mathbf{H}_{0}\right)
$$

exists and are unitary.

vi) The scattering matrix

$$
S\left(\mathbf{V}_{\varepsilon}\right)=\left(W_{\varepsilon}^{+}\right)^{*} W_{\varepsilon}^{-}
$$

can be computed as

$$
S\left(\mathbf{V}_{\varepsilon}\right)=S-\lim _{T, T^{\prime} \rightarrow \infty} \exp \left(i T \mathbf{H}_{0}\right) U_{\varepsilon}\left(T,-T^{\prime}\right) \exp \left(i T^{\prime} \mathbf{H}_{0}\right)
$$

Proof of Theorem $3 i$ and ii). Use Young, Hausdorff-Young inequalities, and the fact that the integral kernels of $\left|\mathbf{H}_{0}\right|^{-\alpha}$ are essentially Bessel potentials $G_{\alpha}(x)$ and then

$$
G_{\alpha} \in L^{1}\left(\mathbb{R}^{3}\right) \cap L^{q}\left(\mathbb{R}^{3}\right) \text { for } 1 \leqq q<3 /(3-\alpha) .
$$

The $\mathbf{H}_{0}$-compactness follows from (3.5) (see the proof of Lemma 5.1 in [21]). iii) follows from i) and the Rellich-Kato theorem [14]. For iv) see [14]. The proof of v) is identical with Dollard's proof for the Schrödinger case [15]. The last point follows from the definition of $S\left(\mathbf{V}_{\varepsilon}\right)$ and the fact that $W_{\varepsilon}^{ \pm}$are unitary.

Our main results are contained in the following 
Theorem 4. Besides the conditions of Theorem 3, suppose that there exist a continuous function $f(s), \lim _{s \rightarrow \pm \infty} f(s)=0$, and a constant $d>0$ such that the spectrum $\Sigma(s)$ of $\mathbf{H}(s)=\mathbf{H}_{0}+\varphi(s) \mathbf{V}$ has the property:

$$
\begin{aligned}
& \Sigma(s)=\Sigma_{+}(s) \cup \Sigma_{-}(s) \\
& \Sigma_{+}(s) \subset[f(s)+d / 2, \infty), \Sigma_{-}(s) \subset(-\infty, f(s)-d / 2] .
\end{aligned}
$$

Then for $\sigma \neq \sigma^{\prime}$

$$
\lim _{\varepsilon \rightarrow 0}\left\|P_{\sigma}^{0} S\left(\mathbf{V}_{\varepsilon}\right) P_{\sigma^{\prime}}^{0}\right\|_{\text {H.S. }}=0 \text {. }
$$

This theorem, combined with Theorem 2, implies that $\mathbf{V}$ is undercritical, which proofs the Conjecture 2.

Remark 3. There exist several proofs of the fact that $P_{\sigma}^{0} S\left(\mathbf{V}_{\varepsilon}\right) P_{\sigma^{\prime}}^{0}$ is HilbertSchmidt, but no one seems to be appropriate for our purpose. Most of them $[5,6$, $9,10]$ are based on the Dyson series which is difficult to control in the adiabatic limit. Another method we are aware of, still requires compact perturbations [19]. Our proof is a generalization of the adiabatic theorem of quantum mechanics $[17$, $20,18]$.

Proof of Theorem 4. A finite number of constants will appear during the proof. For the sake of simplicity, we shall denote all of them by the same letter $c$. They do not depend on $t$ and $\varepsilon$ During the proof, some of the technical points are stated as lemmas which are proved at the end. Note that since $\mathbf{V}$ is $\mathbf{H}_{0}$-compact $\sigma_{\text {ess }}\left(\mathbf{H}_{\varepsilon}\right)=\sigma_{\text {ess }}\left(\mathbf{H}_{0}\right)$ and then $|f(s)| \leqq m-d / 2$.

Lemma 2. Let $P_{\sigma}^{\varepsilon}(t), \sigma= \pm$ be the spectral projection of $\mathbf{H}_{\varepsilon}(t)$ corresponding to $\Sigma_{\sigma}^{\varepsilon}(t) \equiv \Sigma_{\sigma}(\varepsilon t)$. Then

i)

$$
\lim _{T \rightarrow \infty}\left\|P_{\sigma}^{\varepsilon}(T)-P_{\sigma}^{0}\right\|=0 .
$$

ii) $P_{\sigma}^{\varepsilon}(t)$ is norm differentiable as a function of $t$ and

$$
\left\|\frac{d}{d t} P_{\sigma}^{\varepsilon}(t)\right\| \leqq c\left|\frac{d}{d t} \varphi(\varepsilon t)\right| .
$$

Using (3.11) and (3.13) one can see that

$$
\begin{aligned}
P_{\sigma}^{0} S\left(\mathbf{V}_{\varepsilon}\right) P_{\sigma^{\prime}}^{0}= & \underset{T, T^{\prime} \rightarrow \infty}{S-\lim } \exp \left(i T \mathbf{H}_{0}\right) P_{\sigma}^{\varepsilon}(T) U_{\varepsilon}\left(T,-T^{\prime}\right) \\
& \cdot P_{\sigma^{\prime}}^{\varepsilon}\left(-T^{\prime}\right) \exp \left(i T^{\prime} \mathbf{H}_{0}\right) .
\end{aligned}
$$

Let $K^{\varepsilon}(t)$ be defined by

$$
K^{\varepsilon}(t)=i \sum_{\sigma}\left(\frac{d}{d t} P_{\sigma}^{\varepsilon}(t)\right) P_{\sigma}^{\varepsilon}(t) .
$$




\section{Lemma 3.}

$$
\begin{aligned}
& K^{\varepsilon}(t)^{*}=K^{\varepsilon}(t), \\
& {\left[P_{\sigma}^{\varepsilon}(t), K^{\varepsilon}(t)\right]=-i \frac{d}{d t} P_{\sigma}^{\varepsilon}(t) .}
\end{aligned}
$$

Let $A^{\varepsilon}\left(t, t_{0}\right)$ be defined by

$$
i \frac{d}{d t} A^{\varepsilon}\left(t, t_{0}\right)=K^{\varepsilon}(t) A^{\varepsilon}\left(t, t_{0}\right) ; A^{\varepsilon}\left(t_{0}, t_{0}\right)=1 .
$$

Equation (3.18) has a unique solution satisfying [14, Theorem X69]

$$
A^{\varepsilon}\left(t, t_{0}\right)^{-1}=A^{\varepsilon}\left(t, t_{0}\right)^{*} .
$$

Using (3.17) and (3.18), one can see that

$$
\frac{d}{d t}\left(A^{\varepsilon}\left(t, t_{0}\right)^{*} P_{\sigma}^{\varepsilon}(t) A^{\varepsilon}\left(t, t_{0}\right)\right)=0
$$

which implies

$$
P_{\sigma}^{\varepsilon}\left(t_{0}\right)=A^{\varepsilon}\left(t, t_{0}\right)^{*} P_{\sigma}^{\varepsilon}(t) A^{\varepsilon}\left(t, t_{0}\right) .
$$

We shall define $\tilde{U}_{\varepsilon}\left(t, t_{0}\right)$ by

$$
\tilde{U}_{\varepsilon}\left(t, t_{0}\right)=A^{\varepsilon}\left(t, t_{0}\right) * U_{\varepsilon}\left(t, t_{0}\right) \text {. }
$$

By direct computation

$$
\begin{aligned}
& i \frac{d}{d t} \tilde{U}_{\varepsilon}\left(t, t_{0}\right)=A^{\varepsilon}\left(t, t_{0}\right)^{*}\left[\mathbf{H}_{\varepsilon}(t)\right. \\
& \left.\quad-K^{\varepsilon}(t)\right] A^{\varepsilon}\left(t, t_{0}\right) \tilde{U}_{\varepsilon}\left(t, t_{0}\right) ; \tilde{U}_{\varepsilon}\left(t_{0}, t_{0}\right)=1 .
\end{aligned}
$$

Clearly $\Sigma\left(A^{\varepsilon}\left(t, t_{0}\right) * \mathbf{H}_{\varepsilon}(t) A^{\varepsilon}\left(t, t_{0}\right)\right)=\Sigma\left(\mathbf{H}_{\varepsilon}(t)\right)$, and by (3.19), $P_{\sigma}^{\varepsilon}\left(t_{0}\right)$ are the spectral projections of $A^{\varepsilon}\left(t, t_{0}\right)^{*} \mathbf{H}_{\varepsilon}(t) A^{\varepsilon}\left(t, t_{0}\right)$ corresponding to $\Sigma_{\sigma}^{\varepsilon}(t)$. By (3.14), (3.15), and (3.3)

$$
\left\|K^{\varepsilon}(t)\right\| \leqq c|\varepsilon|
$$

and then for small enough $|\varepsilon|$

$\Sigma\left(A^{\varepsilon}\left(t, t_{0}\right)^{*}\left[\mathbf{H}_{\varepsilon}(t)-K^{\varepsilon}(t)\right] A^{\varepsilon}\left(t, t_{0}\right)\right)$ has two disjoint components $\tilde{\Sigma}_{\sigma}^{\varepsilon}(t)$ and for all $\lambda \in \tilde{\Sigma}_{\sigma}^{\varepsilon}(t), \operatorname{dist}\left(\lambda, \Sigma_{\sigma}^{\varepsilon}(t)\right) \leqq c|\varepsilon|$.

We shall perform the above procedure once more defining $B^{\varepsilon}\left(t, t_{0}\right)$ by

$$
\begin{aligned}
& i \frac{d}{d t} B^{\varepsilon}\left(t, t_{0}\right)=L^{\varepsilon}(t) B^{\varepsilon}\left(t, t_{0}\right) ; B^{\varepsilon}\left(t_{0}, t_{0}\right)=1, \\
& L^{\varepsilon}(t)=i \sum_{\sigma}\left(\frac{d}{d t} Q_{\sigma}^{\varepsilon}(t)\right) Q_{\sigma}^{\varepsilon}(t),
\end{aligned}
$$

where $Q_{\sigma}^{\varepsilon}(t)$ are the spectral projections of

$$
A^{\varepsilon}\left(t, t_{0}\right)^{*}\left[\mathbf{H}_{\varepsilon}(t)-K^{\varepsilon}(t)\right] A^{\varepsilon}\left(t, t_{0}\right)
$$


corresponding to $\tilde{\Sigma}_{\sigma}^{\varepsilon}(t)$. In order (3.24) to make sense, we need information about $Q_{\sigma}^{\varepsilon}(t)$. The following technical lemma is the heart of the proof

\section{Lemma 4.}

i) $\left\|Q_{\sigma}^{\varepsilon}(t)-P_{\sigma}^{\varepsilon}\left(t_{0}\right)\right\|_{\text {H.s. }} \leqq c\left|\frac{d}{d t} \varphi(\varepsilon t)\right|$.

ii) $Q_{\sigma}^{\varepsilon}(t)$ is norm differentiable and

$$
\left\|\frac{d}{d t} Q_{\sigma}^{\varepsilon}(t)\right\|_{\text {H.S. }} \leqq c\left(\left|\frac{d}{d t} \varphi(\varepsilon t)\right|^{2}+\left|\frac{d^{2}}{d t^{2}} \varphi(\varepsilon t)\right|\right) .
$$

As for $A^{\varepsilon}\left(t, t_{0}\right)$ one has

$$
Q_{\sigma}^{\varepsilon}\left(t_{0}\right)=B^{\varepsilon}\left(t, t_{0}\right)^{*} Q_{\sigma}^{\varepsilon}(t) B^{\varepsilon}\left(t, t_{0}\right) .
$$

Let now $\Phi^{\varepsilon}$ and $\Psi^{\varepsilon}$ be defined by

$$
\begin{aligned}
i \frac{d}{d t} \Phi^{\varepsilon}\left(t, t_{0}\right)= & B^{\varepsilon}\left(t, t_{0}\right)^{*} A^{\varepsilon}\left(t, t_{0}\right) *\left(\mathbf{H}_{\varepsilon}(t)-K^{\varepsilon}(t)\right) \\
& \cdot A^{\varepsilon}\left(t, t_{0}\right) B^{\varepsilon}\left(t, t_{0}\right) \Phi^{\varepsilon}\left(t, t_{0}\right) \\
& \cdot \Phi^{\varepsilon}\left(t_{0}, t_{0}\right)=1 \\
\Psi^{\varepsilon}\left(t, t_{0}\right)=\Phi^{\varepsilon}( & \left.t, t_{0}\right)^{*} B^{\varepsilon}\left(t, t_{0}\right)^{*} \tilde{U}_{\varepsilon}\left(t, t_{0}\right) .
\end{aligned}
$$

By construction

$$
\Phi^{\varepsilon}\left(t, t_{0}\right) Q_{\sigma}^{\varepsilon}\left(t_{0}\right)=Q_{\sigma}^{\varepsilon}\left(t_{0}\right) \Phi^{\varepsilon}\left(t, t_{0}\right)
$$

and

$$
\begin{aligned}
i \frac{d}{d t} \Psi^{\varepsilon}\left(t, t_{0}\right)= & -\Phi^{\varepsilon}\left(t, t_{0}\right)^{*} B^{\varepsilon}\left(t, t_{0}\right)^{*} L^{\varepsilon}(t) B^{\varepsilon}\left(t, t_{0}\right) \Phi^{\varepsilon}\left(t, t_{0}\right) \\
& . \Psi^{\varepsilon}\left(t, t_{0}\right) ; \quad \Psi^{\varepsilon}\left(t_{0}, t_{0}\right)=1 .
\end{aligned}
$$

Writing (3.31) as an integral equation and estimating with help of Lemma 4ii), one obtains

$$
\begin{aligned}
\left\|\Psi^{\varepsilon}\left(t, t_{0}\right)-1\right\|_{\text {H.S. }} \leqq & c \mid \int_{t_{0}}^{t}\left(\left|\frac{d}{d t^{\prime}} \varphi\left(\varepsilon t^{\prime}\right)\right|^{2}\right. \\
& \left.+\left|\frac{d^{2}}{d t^{\prime 2}} \varphi\left(\varepsilon t^{\prime}\right)\right|\right) d t^{\prime} \mid .
\end{aligned}
$$

Using (3.19), (3.20), (3.27), (3.29), and (3.30), one gets the following identity

$$
\begin{aligned}
U_{\varepsilon}( & \left.t, t_{0}\right) P_{\sigma}^{\varepsilon}\left(t_{0}\right)-P_{\sigma}^{\varepsilon}(t) U_{\varepsilon}\left(t, t_{0}\right) \\
= & A^{\varepsilon}\left(t, t_{0}\right) B^{\varepsilon}\left(t, t_{0}\right) \Phi^{\varepsilon}\left(t, t_{0}\right)\left[\Psi^{\varepsilon}\left(t, t_{0}\right)-1\right] P_{\sigma}^{\varepsilon}\left(t_{0}\right) \\
& +A^{\varepsilon}\left(t, t_{0}\right) B^{\varepsilon}\left(t, t_{0}\right) \Phi^{\varepsilon}\left(t, t_{0}\right)\left[P_{\sigma}^{\varepsilon}\left(t_{0}\right)-Q_{\sigma}^{\varepsilon}\left(t_{0}\right)\right] \\
& +A^{\varepsilon}\left(t, t_{0}\right)\left[Q_{\sigma}^{\varepsilon}(t)-P_{\sigma}^{\varepsilon}\left(t_{0}\right)\right] B^{\varepsilon}\left(t, t_{0}\right) \Phi^{\varepsilon}\left(t, t_{0}\right) \\
& +P_{\sigma}^{\varepsilon}(t) A^{\varepsilon}\left(t, t_{0}\right) B^{\varepsilon}\left(t, t_{0}\right) \Phi^{\varepsilon}\left(t, t_{0}\right)\left[1-\Psi^{\varepsilon}\left(t, t_{0}\right)\right] .
\end{aligned}
$$


From (3.33) and Lemma 4, one obtains the final estimate for $\sigma \neq \sigma^{\prime}$ and $t, t_{0} \in \mathbb{R}$

$$
\begin{aligned}
& \left\|P_{\sigma}^{\varepsilon}(t) U_{\varepsilon}\left(t, t_{0}\right) P_{\sigma^{\prime}}^{\varepsilon}\left(t_{0}\right)\right\|_{\text {H.S. }} \\
& \leqq \\
& \quad c\left(\left|\frac{d}{d t} \varphi(\varepsilon t)\right|+\left|\frac{d}{d t} \varphi(\varepsilon t)\right|_{t=t_{0}}\right. \\
& \left.\quad+\left|\int_{t_{0}}^{t}\left(\left|\frac{d}{d t^{\prime}} \varphi\left(\varepsilon t^{\prime}\right)\right|^{2}+\left|\frac{d^{2}}{d t^{\prime 2}} \varphi\left(\varepsilon t^{\prime}\right)\right|\right) d t^{\prime}\right|\right) .
\end{aligned}
$$

Lemma 5. Let $A_{n}$ be a strongly convergent sequence of Hilbert-Schmidt operators in a separable Hilbert space, $\left\|A_{n}\right\|_{\text {H.S. }} \leqq c_{n}, c=\varlimsup \lim c_{n}<\infty$ and $A=s-\lim A_{n}$. Then $A$ is Hilbert-Schmidt and $\|A\|_{\text {H.S. }} \leqq c$.

From $\left(3.11^{\prime}\right),(3.34)$, and Lemma 5, it follows that

$$
\left\|P_{\sigma}^{0} S\left(\mathbf{V}_{\varepsilon}\right) P_{\sigma^{\prime}}^{0}\right\|_{\text {H.S. }} \leqq c \int_{-\infty}^{\infty}\left(\left|\frac{d}{d t} \varphi(\varepsilon t)\right|^{2}+\left|\frac{d^{2}}{d t^{2}} \varphi(\varepsilon t)\right|\right) d t
$$

which together with (3.6) finishes the proof of the theorem.

Proof of Lemma 2. Let $R_{t}^{\varepsilon}(z)=\left(\mathbf{H}_{\varepsilon}(t)-z\right)^{-1}, R_{0}(z)=\left(\mathbf{H}_{0}-z\right)^{-1}$ be the resolvents of $\mathbf{H}_{\varepsilon}(t)$ and $\mathbf{H}_{0}$ respectively. We shall denote by $x$ the midpoint of the gap between $\Sigma_{+}^{\varepsilon}(t)$ and $\Sigma_{-}^{\varepsilon}(t)$. Note that $\operatorname{dist}(x,\{ \pm m\}) \geqq d / 2$.

Using the integral representation of the spectral projections, and the resolvent equation

$$
R_{t}^{\varepsilon}(z)-R_{0}(z)=-\varphi(\varepsilon t) R_{0}(z) \mathbf{V} R_{t}^{\varepsilon}(z)
$$

one obtains

$$
P_{\sigma}^{\varepsilon}(t)-P_{\sigma}^{0}=-\sigma \frac{\varphi(\varepsilon t)}{2 \pi} \int_{-\infty}^{\infty} R_{0}(x+i y) \mathbf{V} R_{t}^{\varepsilon}(x+i y) d y .
$$

By the functional calculus for self-adjoint operators one has for

$$
\begin{aligned}
& 0 \leqq \delta \leqq 1 \\
& \left\|\left|\mathbf{H}_{0} R_{0}(x+i y)\right|^{1-\delta}\right\| \leqq c .
\end{aligned}
$$

Taking $\delta=\frac{p-3}{2 p}$ and combining Theorem 3i) and (3.38) one obtains

$$
\begin{aligned}
& \left\|R_{0}(x+i y) \mathbf{V} R_{t}^{\varepsilon}(x+i y)\right\| \\
& \leqq \\
& \qquad\left|\mathbf{H}_{0}\right|^{-\frac{p+3}{2 p}} \mathbf{V}\|\|\left|\mathbf{H}_{0} R_{0}(x+i y)\right|^{(p+3) / 2 p} \| \\
& \quad \cdot\left\|R_{t}^{\varepsilon}(x+i y)\right\|\left\|R_{0}(x+i y)\right\|^{(p-3) / 2 p} \\
& \leqq c\left(y^{2}+d^{2} / 4\right)^{-3(p-1) / 4 p}
\end{aligned}
$$

which together with (3.37) and the definition of $\varphi(s)$ finishes the proof of (3.13).

We remark now that $R_{t}^{\varepsilon}(z)$ is norm differentiable and

$$
\frac{d}{d t} R_{t}^{\varepsilon}(z)=-\left(\frac{d}{d t} \varphi(\varepsilon t)\right) R_{t}^{\varepsilon}(z) \mathbf{V} R_{t}^{\varepsilon}(z)
$$


Differentiating (3.37) with respect to $t$ and using (3.39), one has

$$
\begin{aligned}
& \frac{d}{d t} P_{\sigma}^{\varepsilon}(t)=-\frac{\sigma}{2 \pi}\left(\frac{d}{d t} \varphi(\varepsilon t)\right) \\
& \cdot \int_{-\infty}^{\infty} R_{0}(x+i y) \mathbf{V} R_{t}^{\varepsilon}(x+i y) d y \\
& +\frac{\sigma}{2 \pi} \varphi(\varepsilon t)\left(\frac{d}{d t} \varphi(\varepsilon t)\right) \\
& \cdot \int_{-\infty}^{\infty} R_{0}(x+i y) \mathbf{V} R_{t}^{\varepsilon}(x+i y) \mathbf{V} R_{t}^{\varepsilon}(x+i y) d y .
\end{aligned}
$$

From (3.6) it follows that there exists $0 \leqq y_{0}<\infty$ such that $\left\|\mathbf{V} R_{0}\left(x+i y_{0}\right)\right\|<1 / 2$ for all $x \in[-m+d / 2, m-d / 2]$.

Then

$$
\begin{aligned}
& R_{t}^{\varepsilon}\left(x+i y_{0}\right)=R_{0}\left(x+i y_{0}\right) \\
& \quad-R_{0}\left(x+i y_{0}\right)\left[1+\varphi(\varepsilon t) \vee R_{0}\left(x+i y_{0}\right)\right]^{-1} .
\end{aligned}
$$

Moreover from the functional calculus

$$
\sup _{x \in[-m+d / 2, m-d / 2]}\left\|\left(\mathbf{H}_{\varepsilon}(t)-x-i y_{0}\right) R_{t}^{\varepsilon}(x+i y)\right\| \leqq c .
$$

Combining (3.41) and (3.42) one obtains

$$
\left\|\mathbf{V} R_{t}^{\varepsilon}(x+i y)\right\| \leqq c .
$$

From (3.40) and (3.43) one has

$$
\begin{aligned}
& \left\|\frac{d}{d t} P_{\sigma}^{\varepsilon}(t)\right\| \leqq c\left|\frac{d}{d t} \varphi(\varepsilon t)\right| \\
& \cdot \int_{-\infty}^{\infty}\left\|R_{0}(x+i y) \mathbf{V} R_{t}^{\varepsilon}(x+i y)\right\| d y
\end{aligned}
$$

and the integral on the r.h.s. of (3.44) has been estimated in the proof of (3.13). This finishes the proof of Lemma 2.

Proof of Lemma 3. See [20].

Proof of Lemma 4. Let $\tilde{R}_{t}^{\varepsilon}(z)=\left(\mathbf{H}_{\varepsilon}(t)-K^{\varepsilon}(t)-z\right)^{-1}$.

Noting that $P_{\sigma}^{\varepsilon}\left(t_{0}\right)$ are the spectral projection of

$$
A^{\varepsilon}\left(t, t_{0}\right) * \mathbf{H}_{\varepsilon}(t) A^{\varepsilon}\left(t, t_{0}\right)
$$

for all $t$ one can write

$$
\begin{aligned}
Q_{\sigma}^{\varepsilon}(t) & -P_{\sigma}^{\varepsilon}\left(t_{0}\right) \\
= & \frac{1}{2 \pi} \int_{-\infty}^{\infty}\left(A^{\varepsilon}\left(t, t_{0}\right)^{*} \mathbf{H}_{\varepsilon}(t) A^{\varepsilon}\left(t, t_{0}\right)-x-i y\right)^{-1} \\
& \cdot A^{\varepsilon}\left(t, t_{0}\right)^{*} K^{\varepsilon}(t) A^{\varepsilon}\left(t, t_{0}\right)
\end{aligned}
$$




$$
\begin{aligned}
& \cdot\left(A^{\varepsilon}\left(t, t_{0}\right)^{*}\left(\mathbf{H}_{\varepsilon}(t)-K^{\varepsilon}(t)\right) A^{\varepsilon}\left(t, t_{0}\right)-x-i y\right)^{-1} \\
= & \frac{1}{2 \pi} A^{\varepsilon}\left(t, t_{0}\right)^{*}\left[\int_{-\infty}^{\infty} R_{t}^{\varepsilon}(x+i y) \cdot K^{\varepsilon}(t) \tilde{R}_{t}^{\varepsilon}(x+i y) d y\right] A^{\varepsilon}\left(t, t_{0}\right) .
\end{aligned}
$$

Combining (3.15), (3.45) and the following form of (3.40)

$$
\frac{d}{d t} P_{\sigma}^{\varepsilon}(t)=-\frac{\sigma}{2 \pi}\left(\frac{d}{d t} \varphi(\varepsilon t)\right) \int_{-\infty}^{\infty} R_{t}^{\varepsilon}(x+i y) \mathbf{V} R_{t}^{\varepsilon}(x+i y) d y
$$

one gets

$$
\begin{aligned}
& Q_{\sigma}^{\varepsilon}(t)-P_{\sigma}^{\varepsilon}\left(t_{0}\right) \\
& =-\frac{i \sigma}{4 \pi^{2}}\left(\frac{d}{d t} \varphi(\varepsilon t)\right) A^{\varepsilon}\left(t, t_{0}\right)^{*} \cdot\left[\sum_{\sigma^{\prime}} \int_{-\infty}^{\infty} \int_{-\infty} d y d v R_{t}^{\varepsilon}(x+i y) R_{t}^{\varepsilon}(x+i v) \mathbf{V}\right. \\
& \left.\quad \cdot R_{t}^{\varepsilon}(x+i v) P_{\sigma^{\prime}}^{\varepsilon}(t) \tilde{R}_{t}^{\varepsilon}(x+i y)\right] A^{\varepsilon}\left(t, t_{0}\right) .
\end{aligned}
$$

The essential property of this formula (3.46) is the appearance of the operator $R_{t}^{\varepsilon}(x+i y) R_{t}^{\varepsilon}(x+i v) \mathbf{V}$ and of $\frac{d}{d t} \varphi(\varepsilon t)$ in front.

In fact, one can see by inspection that (3.25) is proved if

$$
\begin{aligned}
& \left\|R_{t}^{\varepsilon}(x+i y) R_{t}^{\varepsilon}(x+i v) \mathbf{V}\right\|_{\text {H.S. }} \\
& \quad \leqq c\left[\left(y^{2}+d^{2} / 4\right)\left(v^{2}+d^{2} / 4\right)\right]^{-1 / 16} .
\end{aligned}
$$

Inserting

$$
R_{t}^{\varepsilon}(z)=R_{0}(z)-\varphi(\varepsilon t) R_{0}(z) \mathbf{V} R_{0}(z)+\varphi^{2}(\varepsilon t) R_{0}(z) \mathbf{V} R_{t}^{\varepsilon}(z) \mathbf{V} R_{0}(z)
$$

in (3.47), one sees that all the resulting terms contain $R_{0}(x+i y) R_{0}(x+i v) \mathbf{V}$. Taking into account (3.6) and (3.43), the only thing we have to prove is

$$
\left\|R_{0}(x+i y) R_{0}(x+i v) \mathbf{V}\right\|_{\text {H.S. }} \leqq c\left[\left(y^{2}+d^{2} / 4\right)\left(v^{2}+d^{2} / 4\right)\right]^{-1 / 16}
$$

which follows from (3.7) and (3.38) taking $\alpha=7 / 4$ and $\delta=1 / 8$.

This finishes the proof of Lemma 4i).

For the proof of Lemma 4ii), let us examine the terms one gets by differentiating the r.h.s. of (3.45). (Warning: the contour of integration in the spectral projection integral formula is kept fixed during the differentiation.) Taking into account (3.39) and

$$
\frac{d}{d t} \tilde{R}_{t}^{\varepsilon}(z)=-\tilde{R}_{t}^{\varepsilon}(z)\left[\left(\frac{d}{d t} \varphi(\varepsilon t)\right) \mathbf{V}-\frac{d}{d t} K^{\varepsilon}(t)\right] \tilde{R}_{t}^{\varepsilon}(z)
$$

one observes that

1. All terms contain at last either $\left(\frac{d}{d t} \varphi(\varepsilon t)\right)^{2}$ or $\frac{d^{2}}{d t^{2}} \varphi(\varepsilon t)$.

2. Writing the integral formula for $\frac{d}{d t} P_{\sigma}^{\varepsilon}(t)$ or $\frac{d^{2}}{d t^{2}} P_{\sigma}^{\varepsilon}(t)$ which appear in $K^{\varepsilon}(t)$ and $\frac{d}{d t} K^{\varepsilon}(t)$ respectively, the factor $R_{t}^{\varepsilon}(x+i y) R_{t}^{\varepsilon}(x+i v) \mathbf{V}$ arises. Then all the terms 
can be estimated as in the proof of Lemma 4i). Note that the norm of $V \tilde{R}_{t}^{\varepsilon}(z)$ can be easily controled using

$$
\tilde{R}_{t}^{\varepsilon}(z)=R_{t}^{\varepsilon}(z)-R_{t}^{\varepsilon}(z) K^{\varepsilon}(t) \tilde{R}_{t}^{\varepsilon}(z) .
$$

This finishes the proof of Lemma 4.

Proof of Lemma 5. See [22, Chap. V, Theorem 15] and the fact that for a finite rank operator $F$,

$$
\lim _{n \rightarrow \infty} \operatorname{Tr}\left(A_{n} F\right)=\operatorname{Tr}(A F) .
$$

Acknowledgements. It is a pleasure to thank Prof. G. Scharf for long and fruitful discussions. I am grateful to the unnamed referee for pointing me the argument contained in Lemma 5 . The original argument needs an additional condition on $\varphi(s)$, namely $\int_{-\infty}^{\infty} \varphi(s) d s<\infty$, but in this case, a stronger result is obtained

$$
P_{\sigma}^{0} S\left(\mathbf{V}_{\varepsilon}\right) P_{\sigma^{\prime}}^{0}=\text { H.S.- } \lim _{T, T^{\prime} \rightarrow \infty} \exp \left(i T \mathbf{H}_{0}\right) P_{\sigma}^{\varepsilon}(T) U_{\varepsilon}\left(T,-T^{\prime}\right) \cdot P_{\sigma^{\prime}}^{\varepsilon}\left(-T^{\prime}\right) \exp \left(i T^{\prime} \mathbf{H}_{0}\right)
$$

\section{References}

1. Zeldovich, Ya.B., Popov, V.S.: Usp. Fiz. Nauk 105, 403-440 (1971)

2. Reinhardt, J., Greiner, W.: Rep. Prog. Phys. 40, 219-295 (1977)

3. Rafelski, J., Fulcher, L.P., Klein, A.: Phys. Rep. 38C (1978)

4. Brodsky, S.J., Mohr, P.K.: Q.E.D. in strong and supercritical fields in structure and collisions of ions and atoms (ed. I. A.Sellin). Berlin, Heidelberg, New York: Springer 1978

5. Seiler, R.: Particles with spin $s \leqq 1$ in an external field. In : Lecture notes in physics, Vol. 73. Berlin, Heidelberg, New York: Springer 1978

6. Wightman, A.S.: Invariant wave equations; general theory and applications to the external field problem. In: Lecture notes in physics, Vol. 73. Berlin, Heidelberg, New York: Springer 1978

7. Klaus, M., Scharf, G. : Helv. Phys. Acta 50, 779-802 (1978); 50, 803, 814 (1978)

8. Nenciu, G., Scharf, G.: Helv. Phys. Acta 51, 412-424 (1978)

9. Palmer, J.: J. Math. Anal. Appl. 64, 189-215 (1978)

10. Rujsenaars, S.N.M.: J. Funct. Analysis 33, 47-57 (1979)

11. Nenciu, G.: Strong external fields in Q.E.D. rigorous results. Proceedings of the International Summer School in Heavy Ion Physics. Predeal Rumania 1978

12. Bongaarts, P.J.M.: Ann. Phys. (N.Y.) 56, 108-139 (1970)

13. Matthews, P.T., Salam, A.: Phys. Rev. 90, 690-695 (1953)

14. Reed, M., Simon, B.: Methods of modern mathematical physics, Vol. II. New York: Academic Press 1975

15. Dollard, J.D. : J. Math. Phys. (N.Y.) 7, 802-811 (1966)

16. Berezin, F.A.: The method of second quantization. New York, London: Academic Press 1966

17. Kato, T.: J. Phys. Soc. Jpn. 5, 435-439 (1950)

18. Nenciu, G.: J. Phys. A13, L15-L18 (1980)

19. Wald, R.M.: Ann. Phys. (N.Y.) 118, 490-510 (1979)

20. Messiah, A.: Mécanique quantique, Tome II. Paris: Dunod 1960

21. Nenciu, G.: Commun. Math. Phys. 48, 235-247 (1976)

22. Schatten, R. : Norm ideals of completely continuous operators, 2nd printing. Berlin, Heidelberg, New York: Springer 1970

Communicated by B. Simon

Received November 27, 1979; in revised form March 4, 1980 